\title{
Desempenho no Jogo, Estratégias de Aprendizagem e Compreensão na Leitura ${ }^{1}$
}

\author{
Maria Aparecida Mezzalira Gomes ${ }^{2}$ \\ Centro Universitário Padre Anchieta e \\ Escola Superior de Educação Física de Jundiaí \\ Evely Boruchovitch \\ Universidade Estadual de Campinas
}

\begin{abstract}
RESUMO - Considerando a escassez de instrumentos nacionais de avaliação psicoeducacional e a importância das estratégias de aprendizagem na escolarização formal, este estudo objetivou: a) analisar o potencial do jogo Bingo Melhor Estudante, adaptado para avaliar as percepções das características de um bom estudante, entre 29 alunos de $4^{a}$ série do ensino fundamental de uma escola pública; b) verificar as relações entre o desempenho dos participantes no jogo, num teste de compreensão em leitura e numa escala de estratégias de aprendizagem. Os dados foram coletados mediante o jogo, o teste de compreensão de leitura e a escala e, analisados qualitativa e quantitativamente. O jogo parece útil para a avaliação das percepções das características de um bom estudante. Correlações significativas foram encontradas entre o desempenho no jogo, na escala e no Cloze. Os dados são discutidos à luz da Psicologia Cognitiva baseada na Teoria do Processamento da Informação.
\end{abstract}

Palavras-chave: jogo de bingo; estratégias de aprendizagem; compreensão da leitura; processamento de informação.

\section{Achievement in a Game, Learning Strategies and Reading Comprehension}

\begin{abstract}
Considering the lack of national instruments of psychoeducational evaluation and the importance of learning strategies during formal education, this study had the following objectives: a) to analyze the potential of Bingo The Best Student, an adapted game, to evaluate pupils' perceptions of the characteristics of a good student, among 29 fourth grade students of a public school, and b) to examine relationships between participants' achievement in the game, in a Cloze test, and in a learning strategies scale. Data was collected through a game session, an administration of both a Cloze test and a learning strategy scale. Results were analyzed qualitatively and quantitatively. It seems that the adapted game is useful to evaluate pupils' perceptions of characteristics of a good student. Significant correlations were found between participants' achievement in the game, in the scale and in cloze. Data is discussed within the cognitive psychology based on information processing theory framework.
\end{abstract}

Key words: bingo game; learning strategies; reading comprehension; information process.

Estudos recentes divulgados por diversos pesquisadores confirmam os benefícios de intervenções pedagógicas e psicopedagógicas voltadas para o desenvolvimento cognitivo como forma de se evitar o fracasso escolar (Almeida, 2002; Boruchovitch, 1999; Guthrie, Wigfield \& Vonsecker, 2000; Pressley \& Woloshyn,1995; Sadler, 2001; Vauras, Kinnunen \& Rauhanummi,1999). A Psicologia Cognitiva, baseada na Teoria do Processamento de Informação, destaca a importância de uma prática pedagógica que leve em consideração o ensino de estratégias cognitivas e metacognitivas, conteúdos processuais e condicionais, junto com os conhecimentos declarativos, mais privilegiados pelos professores, tendo em vista a promoção da aprendizagem auto-regulada, entre os estudantes, desde o início da escolarização formal (Boruchovitch 2004b; Dembo, 2000; Hacker, 1998; Hong \& O’Neil,

1 As autoras agradecem o apoio financeiro do CNPq (Processo ${ }^{\circ}$ 300162/95-2), bem como as valiosas críticas e sugestões de revisores anônimos.

2 Endereço: Rua Barão de Teffé, 619. Jundiaí, SP, Brasil 13208-761. E-mail:cidgom@uol.com.br
2001; Pozo, 1996; Symons, Snyder, Cariglia-Bull \& Pressley, 1989; Woolfolk, 2000).

Nas condições atuais, em que a universalização do ensino básico é uma necessidade e não mais um ideal a ser atingido, os desafios que se colocam ao educador são imensos. É preciso, antes de tudo, acreditar na potencialidade de aprendizagem dos educandos. Uma nova visão dos processos cognitivos, aliada a uma nova concepção de aprendizagem, permitirá a necessária "diferenciação cognitiva progressiva", segundo a qual um aluno com melhores professores, metodologias de ensino mais adequadas, métodos de estudo mais eficazes, maior motivação e expectativas de sucesso, virá a apresentar, no futuro, melhor desempenho. Deste modo, é possível pretender mudar o destino precoce de fracasso presumido para muitos estudantes considerados "carentes" ou simplesmente "diferentes" (Almeida 1992, 2002).

Segundo Boruchovitch (1999), as estratégias de aprendizagem podem ser ensinadas para alunos de baixo rendimento escolar. Isso pode produzir tanto a melhoria imediata no uso das estratégias envolvidas como no rendimento escolar geral. No entanto, conhecer as estratégias não é suficiente: é preciso saber como e quando usá-las, bem como garantir um estado 
emocional interno satisfatório para que o estudante se engaje em comportamento estratégico. Variáveis psicológicas como o autoconceito, o sentimento de auto-eficácia, a ansiedade, a motivação e as atribuições de causalidade, entre outras, muito se relacionam ao uso apropriado das estratégias de aprendizagem (Bzuneck, 1999, 2001; Da Silva \& Sá, 1993; Valle Arias, González Cabanach, Rodriguez Martinez, Piñeiro Aguín \& Suárez Riveiro, 1999).

Nisbett, Schucksmith e Dansereau (citados por Pozo, 1996) definem as estratégias de aprendizagem como seqüências de procedimentos ou atividades que se escolhem com o propósito de facilitar a aquisição, o armazenamento e/ou a utilização da informação. Têm um caráter consciente e intencional e, em nível mais específico, as estratégias de aprendizagem podem ser consideradas procedimentos adotados para a realização de uma determinada tarefa (Da Silva \& Sá, 1993), como ferramentas auxiliares da aprendizagem.

Alguns teóricos diferenciam as estratégias cognitivas das metacognitivas (Dembo, 1994; Garner \& Alexander, 1989). Para esses autores, enquanto as estratégias cognitivas se referem a comportamentos e pensamentos que influenciam o processo de aprendizagem de maneira que a informação possa ser armazenada mais eficientemente, as estratégias metacognitivas são procedimentos que o indivíduo usa para planejar, monitorar e regular o seu próprio pensamento, estados afetivos e comportamento.

A auto-regulação da aprendizagem tem sido objeto de investigação recente, e assume importância nos atuais estudos sobre aprendizagem (Couceiro Figueira, 1997; Dembo, 2000; Hacker, 1998; Hong \& O’Neil, 2001). As estratégias cognitivas e metacognitivas são instrumentos de auto-regulação, na medida em que contribuem para o estudante promover o próprio processo de aprendizagem, a regulação do próprio pensamento e a manutenção de um estado interno afetivo e motivacional que facilite a aprendizagem (Boruchovitch, 2001a, 2004a; Gomes, 2002). O conceito de aprendizagem auto-regulada aplica-se a conteúdos específicos do currículo. Por esse motivo, destaca-se, no presente estudo, a importância de se desenvolver nos alunos a compreensão auto-regulada em leitura.

Ler não é apenas um processo de pronunciar o texto, mas é uma atividade complexa que envolve raciocínio, ou seja, ler é compreender. Ter competência em leitura significa possuir um repertório de procedimentos estratégicos, saber gerenciar de forma adequada a sua utilização e aplicá-los de modo flexível, em cada situação (Solé, 1992/1998; Vaz, 1998). Leitores proficientes utilizam diferentes estratégias para compreender e interpretar textos. O leitor competente assume o controle e auto-regulação da sua própria leitura. Isso implica, inicialmente, ler com objetivos definidos; a seguir, construir hipóteses sobre o conteúdo da leitura, baseando-se nos seus conhecimentos prévios de mundo e como leitor (Dembo, 2000; Hacker, 1998; Pressley \& Woloshyn, 1995).

Os objetivos que o leitor se propõe alcançar determinam as estratégias a serem utilizadas para a compreensão, ativam o controle sobre o que se compreende, o quanto se compreende e quando não se compreende. Trata-se, pois, do processo de metacognição utilizado para desfazer obstáculos que impedem a compreensão do texto no decorrer da leitura. Ao surgir algum problema, o leitor se dá conta disso e pode dedicar sua atenção a resolvê-lo por meio de estratégias adequadas (Solé, 1992/1998). O ensino e a utilização de estratégias efetivas de leitura são necessários para garantir ao estudante o sucesso acadêmico e profissional (Boruchovitch, 2001a; Gomes, 2002).

O jogo tem sido utilizado no contexto pedagógico e psicopedagógico em atividades de diagnóstico e de intervenção, tendo em vista as possibilidades de promover, por meio dele, o desenvolvimento e a aprendizagem. Brenelli (2001) defende a utilização do jogo como um instrumento diagnóstico, sobretudo com crianças que estão no início do Ensino Fundamental. O êxito, porém, dessas atividades depende do modo como é conduzido o processo de intervenção como afirmam Macedo, Petty e Passos (2000). Quando há envolvimento do participante, o jogo é uma situação privilegiada para a consciência dos aspectos que demandam correções, o que acarretará, cedo ou tarde, uma superação da fase anterior em que a criança se encontrava.

Na aprendizagem em geral e, de um modo específico na leitura, os estudantes poderão ser beneficiados pela utilização de estratégias cognitivas e metacognitivas para obter melhores resultados na ação de aprender. $\mathrm{O}$ mesmo acontece nos jogos de regras, que desenvolvem o pensamento e os processos metacognitivos pela necessidade de se construírem estratégias que conduzam aos objetivos e vencerem os desafios propostos pelo jogo (Gomes, 2002; Gomes \& Boruchovitch, 2004a). Assim, considerando a escassez de instrumentos nacionais de avaliação psicoeducacional à luz da Psicologia Cognitiva baseada na teoria do Processamento da Informação, bem como a importância das estratégias de aprendizagem na escolarização formal, o presente estudo relata resultados de uma pesquisa descritiva e correlacional que teve como eixo os conceitos de estratégias de aprendizagem auto-regulada e de compreensão auto-regulada em leitura.

Mais precisamente, objetivou-se primeiramente analisar o potencial de um jogo, o Bingo Melhor Estudante, adaptado para avaliar a percepção dos alunos de $4^{\mathrm{a}}$ série do Ensino Fundamental acerca das características de um bom estudante. Em segundo lugar, verificar as relações entre o desempenho dos participantes no jogo, num teste de compreensão em leitura e numa escala de estratégias de aprendizagem.

\section{Método}

\section{Participantes}

A amostra foi composta de 29 estudantes, de uma das quatro classes de $4^{\mathrm{a}}$ série do Ensino Fundamental, selecionadas aleatoriamente, de uma escola pública, do Município de Jundiaí, no estado de São Paulo. A faixa etária dos participantes variou de 10 a 12 anos, com uma média de idade de 10 anos e 4 meses. Os participantes eram de ambos os sexos, sendo 18 do sexo masculino (62\%), e 11 do sexo feminino (38\%) e de nível sócio-econômico desfavorecido.

\section{Instrumentos}

Na coleta de dados foram utilizados os seguintes instrumentos: a escala de estratégias de aprendizagem elaborada por Boruchovitch e Santos em 2001 e utilizada por Gomes 
(2002), o Teste de Cloze do texto "O gato e o rato", o jogo Bingo Melhor Estudante, adaptado por Gomes (2002), especialmente para o estudo, e o formulário de avaliação da metacognição de autoria de Gomes e Boruchovitch (Gomes, 2002). A seguir, cada instrumento será apresentado em detalhes, na ordem que foi utilizado no presente estudo.

\section{Escala de estratégias de aprendizagem}

A versão infantil dessa escala consta de 40 questões do tipo escala likert, com três alternativas (sempre, algumas vezes e nunca) e uma questão aberta na qual se avalia se o estudante menciona usar alguma estratégia não prevista pelos autores. Os itens da escala foram construídos, levando-se em conta a taxionomia de estratégias de aprendizagem de Mckeachie, Pintrich, Lin, Smith e Sharma (citados por Dembo, 1994), de forma a contemplar as principais estratégias de aprendizagem cognitivas (ensaio, elaboração e organização) e metacognitivas (planejamento, monitoramento, controle e a regulação da aprendizagem), tanto para tarefas simples de aprendizagem quanto para as complexas. A alternativa sempre valeu dois pontos, algumas vezes foi pontuada com um ponto e a alternativa nunca foi pontuada com zero ponto, com exceção de nove questões, que apresentam essa pontuação invertida, devido à redação dos itens. O valor máximo possível de pontos obtidos na escala foi de 80 pontos. A escala pode ser aplicada de forma individual ou coletiva. A título de ilustração, um exemplo de uma questão dessa escala é: "Você tenta descobrir quais as idéias principais do texto que está lendo ou estudando" (questão 13).

Para averiguar a consistência interna da escala, 443 participantes de $3^{\mathrm{a}}$, $4^{\mathrm{a}}$ e $5^{\mathrm{a}}$ séries de escolas públicas do ensino fundamental responderam o instrumento, tendo-se obtido um Alpha de Cronbach no valor de 0.74 , indicando que a escala apresenta um índice aceitável de consistência interna (Boruchovitch, 2004b).

\section{Teste Cloze}

O teste Cloze, técnica de complementação de textos, de origem anglo-saxônica, permite ao mesmo tempo, a avaliação e o desenvolvimento da compreensão em leitura; substitui com vantagens as tradicionais perguntas de interpretação que, muitas vezes, sugerem ao sujeito a resposta esperada. O texto utilizado no presente estudo foi "O gato e o rato", de Alliende, Condemarín, Chadwick e Milicic (1990/1994). Esse instrumento conta com 25 lacunas, num conjunto de 182 palavras. De acordo com os autores, a pontuação se dá pelo cálculo da porcentagem de acertos, com correção literal quando se trata de aferição dos resultados, sendo que, abaixo de $44 \%$ de acertos indicam um nível de frustração (pouca fluência e compreensão); entre 44 e 57\% de acertos indicam um "nível de instrução" (leitura satisfatória) e, acima de 57\%, indicam um "nível independente" de leitura, isto é, leitura fluente, com facilidade e compreensão.

\section{O jogo "Bingo Melhor Estudante"}

O "Bingo Melhor Estudante" (BMG) foi adaptado por Gomes (2002), especialmente para esse estudo. Acredita-se que o instrumento possibilite avaliar a percepção do estudante sobre as características dos bons estudantes como alunos que utilizam estratégias cognitivas e metacognitivas de aprendizagem necessárias à aprendizagem auto-regulada. Mais precisamente, o objetivo do jogo é levar o estudante a confrontar os diferentes procedimentos e reconhecer os que são mais adequados por favorecer as situações de aprendizagem.

Na sua estrutura o BMG é constituído por 20 questões com três alternativas de respostas $(a, b, c)$, que se desdobram, pois, em 60 itens. Na sua dinâmica, assume o caráter de um jogo coletivo bastante popular, o bingo. Para a realização do jogo, o participante deverá ter uma cartela quadriculada e numerada (de um a 20) e o aplicador deverá ter as "Regras do Jogo" e as questões que deverão ser respondidas. Para a realização do presente estudo as questões foram impressas em transparências para serem projetadas em tela ou parede.

Ao invés de marcar na sua cartela os números sorteados, como no jogo tradicional, cada participante deve colocar, no espaço ao lado dos números correspondentes a cada questão, a letra $a, b$ ou $c$, de acordo com a sua percepção sobre a alternativa que corresponde às características de um bom estudante, isto é, de um aluno auto-regulado. Muitas das alternativas propostas nesse jogo ganham sentido em oposição às demais e, por esse motivo, é difícil analisá-las isoladamente, fora do contexto da questão.

Para proceder à adaptação do jogo, inicialmente, foram estudadas as diversas pesquisas divulgadas em diferentes países e, no Brasil, a partir dos conceitos de aprendizagem auto-regulada (Couceiro Figueira, 1997; Hacker, 1998) de estratégias de aprendizagem cognitivas e metacognitivas, que caracterizam o estudante ativo que se percebe como agente de sua própria aprendizagem (Boruchovitch,1999, 2001a, 2001b; Dembo, 2000; Festas, 1998; Hong \& O'Neil, 2001; Pozo, 1996; Solé, 1992/1998) assim como alguns mediadores cognitivos ou crenças metacognitivas (Bzuneck, 1999, 2001; Da Silva \& Sá, 1993; Quintanal Diáz, 1998).

Os 60 itens elaborados foram propostos no jogo de forma tanto a descrever comportamentos dificultadores da aprendizagem ( 24 questões ou $40 \%$ ), quanto os facilitadores (36 questões ou 60\%), entre os quais 12 (20\%) descrevem comportamentos que requerem a metacognição. Entre os itens que se referem às estratégias cognitivas pode-se mencionar o item 17b: "Quando você lê para estudar, faz anotações sobre as idéias principais, para não esquecer?" (estratégia de ensaio); o item 20a: "Antes de começar a ler você presta atenção no título e nas ilustrações, tentando verificar o assunto do texto?" (estratégia de elaboração). Como exemplos de itens que descrevem estratégias metacognitivas, pode-se mencionar o item 3b: "Quando você está fazendo uma prova, uma tarefa de classe ou de casa, relê o que escreveu e corrige se percebe erros". Um dos itens que pode exemplificar alternativas que correspondem a comportamentos dificultadores da aprendizagem é o 5a. "Quando você está na classe, assistindo as aulas você fica olhando para o professor, mas deixa seu pensamento "voar" para longe da sala de aula?".

Para considerar os acertos e estabelecer a pontuação de cada participante, estabeleceu-se como critério os enunciados que correspondiam a procedimentos adaptados, isto é, os comportamentos e decisões facilitadores da aprendizagem dos estudantes, em oposição aos procedimentos inadaptados, 
ou seja, as decisões e os comportamentos dificultadores da aprendizagem dos alunos, em diferentes graus. Cada acerto vale um ponto. $\mathrm{O}$ escore máximo previsto corresponde a 16 pontos, o número de questões de cada cartela, dentre as 20 do total do Bingo. Informações mais detalhadas sobre a adaptação, conteúdo, regras, aplicação, análise do BMG podem ser encontradas em Gomes (2002) e em Gomes e Boruchovitch (2004b).

\section{O formulário de avaliação da metacognição no jogo bingo melhor estudante}

Esse formulário é constituído de quatro questões abertas destinadas a avaliar a percepção dos estudantes sobre a atividade e as relações que estabelecem sobre a aprendizagem. Como exemplo de uma questão desse instrumento, pode se citar: "Que tipo de questões valeu ponto, nesse Bingo diferente"?

\section{Procedimentos}

Inicialmente foi realizado um contato com a Escola durante o qual foram esclarecidos os objetivos do trabalho, junto aos professores, e estabelecido, de comum acordo com eles, o cronograma de atividades. Foram observados os princípios e procedimentos éticos estabelecidos pelo Conselho Nacional de Saúde (CNS, 1996, 1997), tais como o respeito à liberdade e privacidade dos participantes, a não interferência nas rotinas da escola, assim como o caráter confidencial dos dados.

O trabalho de campo desenvolveu-se em três etapas, para a aplicação dos instrumentos utilizados na coleta de dados. Em cada momento houve grande preocupação por parte da pesquisadora, em estabelecer o rapport com os estudantes, promover as condições necessárias para o êxito da atividade, deixando-os à vontade e esclarecendo os objetivos do estudo e de cada procedimento. O preenchimento, pelos alunos, da Escala de Estratégias de Aprendizagem foi realizado individualmente, ou em pequenos grupos, fora da sala de aula, para garantir maior assistência aos participantes. A duração média dessa atividade foi de 30 minutos cada aluno.

A aplicação do teste de compreensão, Cloze, ocorreu em sessão coletiva, após a conversa inicial, a leitura do texto " $\mathrm{O}$ gato e o rato", oralmente pelo pesquisador, e, a seguir, individual e silenciosamente pelos alunos. Depois de recolhidos os textos completos, os participantes receberam o mesmo texto com as lacunas e tiveram o tempo necessário para preenchêlas. A atividade durou 90 minutos.

A sessão do jogo BME foi realizada numa sequiência de diversos procedimentos: a) conversa inicial para estabelecer um clima favorável e para dar os esclarecimentos necessários; b) leitura compartilhada das "Regras do Jogo", com atividades orais destinadas a assegurar a compreensão da atividade; c) distribuição das cartelas para cada um dos participantes, com as devidas explicações sobre a forma de preenchimento; d) projeção e leitura de cada item e suas três alternativas, de forma pausada, para que cada participante pudesse refletir, escolher a resposta desejada e anotá-la na sua cartela, escrevendo a letra correspondente no espaço em branco, ao lado do número da respectiva questão; e) projeção das mesmas questões, porém, apenas com os itens que descrevem os comportamentos favoráveis à aprendizagem, isto é, que correspondem às estratégias de aprendizagem cognitivas e metacognitivas. Nesse momento, os estudantes puderam confrontar as suas respostas com as respostas consideradas corretas. Em caso de acerto, a letra que registraram anteriormente deveria ser circulada; f) contagem dos acertos e, após conferir os resultados, proclamação dos vencedores, isto é, daqueles que tinham conseguido reconhecer as características de um "bom estudante" (estudante auto-regulado); g) conversa, com a turma, sobre o significado do número de pontos obtidos, a relação entre as questões e as atitudes pessoais diante da aprendizagem, bem como os hábitos de estudo, tendo sido salientado que o mais importante era saber distinguir as características de um bom estudante para poder colocar em prática as estratégias aprendidas.

Finalmente, na mesma sessão, foi aplicado o último instrumento, com o propósito de verificar também o automonitoramento dos estudantes relativamente às atividades de jogo, isto é, o grau de metacognição existente e ativado, solicitando-se aos alunos que respondessem às questões do formulário de Avaliação da Metacognição. A aplicação do jogo e as respostas às questões do formulário tiveram a duração de 90 minutos. Cabe mencionar que a coleta de dados do presente estudo foi precedida por um estudo piloto, no qual os instrumentos e procedimentos de coleta de dados foram testados e refinados, quando necessário.

\section{Análise de dados}

A análise dos dados obtidos no presente estudo foi realizada em dois momentos. Primeiramente, foi feito um estudo qualitativo das respostas dos participantes às questões abertas, por meio de análise de conteúdo (Berelson, 1952; Bardin, 1991). Num segundo momento, procedeu-se a uma análise quantitativa dos dados, baseada nos procedimentos de estatística descritiva e inferencial.

A análise qualitativa envolveu o estudo das respostas dos participantes às questões abertas do Formulário de Avaliação Metacognitiva. Dessa forma, pôde-se levar em consideração a percepção deles quanto aos benefícios do BME, tendo em vista o primeiro objetivo do trabalho, que foi a adaptação de um jogo para avaliar a percepção dos estudantes acerca das características de bom estudante.

Para aumentar a confiabilidade da análise de conteúdo realizada, todas as respostas dos participantes foram lidas e foi desenvolvido pelos autores um conjunto de categorias de respostas para cada questão. Cada categoria foi definida operacionalmente e um conjunto de regras para a classificação de uma resposta numa dada categoria foi cuidadosamente elaborado.

A consistência do processo de categorização foi avaliada mediante a utilização de três juízes independentes que analisaram $30 \%$ das respostas dos participantes a cada pergunta. As respostas para essa análise foram selecionadas aleatoriamente e avaliadas pelos juízes de acordo com o conjunto de regras previamente desenvolvido. A porcentagem de correspondência alcançada entre os juízes e os pesquisadores variou de $85 \%$ a $100 \%$ para as três primeiras questões e foi de $100 \%$ para a quarta questão. 
A análise quantitativa envolveu duas etapas. Primeiramente, foram empregados procedimentos de estatística descritiva para avaliar todas as variáveis estudadas em termos de distribuição de freqüência, escores, médias e desvio padrão. Em seguida, computou-se o cálculo do coeficiente de Pearson para estimar as correlações entre os resultados obtidos pelos participantes na escala de estratégias de aprendizagem e os resultados obtidos no jogo, bem como entre os resultados no Teste Cloze e o desempenho no jogo. Foram aceitos resultados estatisticamente significativos ao nível de 0,05 ou inferiores.

\section{Resultados}

\section{Análise qualitativa: as percepções dos participantes}

O objetivo da primeira questão do Formulário de Avaliação Metacognitiva era examinar se os alunos relacionavam os pontos assinalados e o sucesso no jogo à identificação das características de um bom estudante (estudante autoregulado).

A análise de conteúdo das respostas nessa questão apontou para a existência de três categorias de resposta: "percepção genérica" (os participantes não percebem a relação entre ganhar pontos e saber as características de um bom estudante), "ausência da percepção da relação" (os participantes atribuem o "ganhar pontos" à obediência às regras do jogo) e "percepção explícita" da relação entre o resultado no jogo e o próprio comportamento enquanto estudante (Tabela 1).

Tabela 1. Percepção dos alunos quanto à pontuação, no jogo.

\begin{tabular}{lcc}
\hline \multicolumn{1}{c}{ Categorias de respostas } & $\begin{array}{c}\text { Número de } \\
\text { respostas }\end{array}$ & $\%$ \\
\hline Ausência de relação. & 10 & 34,48 \\
Percepção genérica. & 10 & 34,48 \\
$\begin{array}{l}\text { Percepção explícita da relação entre o } \\
\text { resultado no jogo e o próprio comportamento } \\
\text { enquanto estudante. }\end{array}$ & 08 & 27,59 \\
Não respondeu. & 01 & 3,45 \\
\hline Total & 29 & 100 \\
\hline
\end{tabular}

As respostas na categoria Ausência de Relação mostram atenção mais à forma do que ao conteúdo das questões propostas pelo jogo. Como exemplo, pode-se mencionar: " $A$ número l". Nesses casos, pode-se considerar que as respostas não demonstraram percepção dos objetivos do jogo, por parte dos estudantes. Entre as respostas que se enquadram na categoria "percepção genérica" encontram-se as que se relacionaram a erros ou acertos do próprio aluno, tais como: "As respostas certas". Já oito alunos responderam de acordo com os objetivos do jogo e fizeram a relação com a sua vida estudantil. São exemplos dessas respostas: "A que fala de um bom estudante", entre outras.

A identificação dos vencedores do jogo foi o alvo da segunda pergunta do formulário e os resultados são apresentados na Tabela 2. De acordo com a Tabela 2, as respostas na
Tabela 2. Percepção dos alunos sobre os vencedores do jogo.

\begin{tabular}{lcc}
\hline \multicolumn{1}{c}{ Categorias de respostas } & $\begin{array}{c}\text { Número de } \\
\text { respostas }\end{array}$ & $\%$ \\
\hline Maior número de pontos. & 20 & 68,97 \\
Cumprimento das regras. & 03 & 10,34 \\
Características de um bom estudante. & 06 & 20,69 \\
\hline Total & 29 & 100 \\
\hline
\end{tabular}

Tabela 3. Autopercepção dos alunos enquanto estudantes.

\begin{tabular}{lcc}
\hline \multicolumn{1}{c}{ Categorias de respostas } & $\begin{array}{c}\text { Número de } \\
\text { respostas }\end{array}$ & \% \\
\hline Bom êxito na escola. & 08 & 27,60 \\
Sucesso no jogo. & 04 & 13,80 \\
$\begin{array}{l}\text { Atribuir-se características de um bom } \\
\text { estudante. }\end{array}$ & 16 & 55,20 \\
Não se perceber como bom estudante. & 01 & 3,40 \\
\hline Total & 28 & 100 \\
\hline
\end{tabular}

categoria "Maior Número de Pontos" se ativeram ao critério quantitativo do funcionamento de um jogo de regras: para vencer é preciso fazer mais pontos, como por exemplo: "Ganhou quem fez 16 pontos". A categoria "Cumprimento de Regras" pode ser exemplificada pela resposta: "Ganhou quem seguiu as regras". Na categoria "Características de um Bom Estudante" enquadram-se as respostas tais como esta: "É o bom estudante".

A terceira questão tinha como objetivo verificar a percepção dos participantes sobre si mesmos, enquanto estudantes, e ao próprio comportamento relativo à aprendizagem, isto é, se houve identificação entre as características do estudante auto-regulado trabalhadas no jogo e a autopercepção. Os resultados foram sintetizados na Tabela 3. Dos 29 participantes, $28(96,5 \%)$ se consideraram como bons estudantes. Como pode ser visto na Tabela 3 , foram identificadas quatro categorias de respostas para explicar essa autopercepção dos alunos enquanto estudantes: bom êxito na escola, sucesso no jogo, características de um bom estudante, não ser um bom estudante.

Entre as respostas dos alunos que se consideram bons alunos por terem êxito na escola podem-se incluir: "Porque tiro notas boas". Entre as respostas que relacionam o considerar-se bom estudante com o fato de terem tido sucesso no jogo pode ser mencionada: "Se eu não fosse um bom estudante, eu não acertaria”. Finalmente, podem ser destacadas as respostas daqueles que se consideram estudiosos e relatam comportamentos de um bom estudante: "Eu presto atenção nas aulas".

A última questão focalizou o gosto pelo jogo. Todos os participantes, isto é, $100 \%$ deram respostas favoráveis, tendo emergido duas categorias de justificativas como mostra a Tabela 4: prazer pela atividade $(51,72 \%)$ e o reconhecimento dos benefícios do jogo $(48,28 \%)$. A maioria opinou que o jogo "élegal". Entre as respostas mais reflexivas podem ser 
Tabela 4. Gosto pelo jogo.

\begin{tabular}{lcc}
\hline \multicolumn{1}{c}{ Categorias de respostas } & $\begin{array}{c}\text { Número de } \\
\text { respostas }\end{array}$ & $\%$ \\
\hline Prazer pela atividade. & 15 & 51,72 \\
Reconhecimento dos benefícios do jogo. & 14 & 48,28 \\
\hline Total & 29 & 100 \\
\hline
\end{tabular}

Tabela 5. Média e desvio padrão da amostra total no que diz respeito ao desempenho na escala de estratégias de aprendizagem, no cloze e no jogo.

\begin{tabular}{lccc}
\hline \multicolumn{1}{c}{ Instrumentos } & $\mathbf{N}$ & Média & $\sigma$ \\
\hline Escala de estratégias & 29 & 56,79 & 6,39 \\
Teste Cloze & 29 & 13,00 & 4,23 \\
Jogo & 29 & 14,10 & 2,48 \\
\hline
\end{tabular}

destacadas as seguintes: "Porque nós aprendemos o que é ser um bom estudante”, "Com o jogo nós podemos saber quais são os hábitos de um bom estudante”.

\section{Análise quantitativa}

A pontuação dos alunos na escala de estratégias de aprendizagem variou de 43 a 60 pontos, a média do grupo foi de 56,79 e o Desvio Padrão 6,39. Os escores obtidos no teste de Cloze tiveram uma amplitude de dois a 19 pontos, com uma média de 13,00 e desvio padrão 4,23. O escore máximo encontrado foi de 16 pontos. Os resultados obtidos pelos participantes no jogo se situam entre 2 e 16 pontos, com a média 14,10 e o desvio padrão 2,48 , como mostra a Tabela 5.

Como pode ser constatado na Tabela 6, o cálculo do coeficiente de Pearson revelou a existência de correlações estatisticamente significativas entre as seguintes variáveis: os resultados obtidos pelos participantes na escala de estratégias de aprendizagem e os resultados obtidos no jogo $(r=0,476$; $\mathrm{p}=0,01)$, bem como entre os resultados no Teste Cloze e o desempenho no jogo, confirmando as expectativas iniciais $(r$ $=0,516 ; p=0,01)$. As correlações encontradas nos dois casos foram, pois, positivas, moderadas e altamente significativas do ponto de vista estatístico. Mais precisamente, pode-se dizer que quanto melhor o desempenho no jogo, maior foi

Tabela 6. Correlação de Pearson entre as variáveis.

\begin{tabular}{lccc}
\hline & $\begin{array}{c}\text { Escala de } \\
\text { Estratégias de } \\
\text { Aprendizagem }\end{array}$ & Cloze & Jogo \\
\hline $\begin{array}{l}\text { Escala de Estratégias de } \\
\text { Aprendizagem. }\end{array}$ & 1,000 & & \\
Cloze & 0,170 & 1,000 & \\
Jogo & $0,476^{* *}$ & $0,516^{* *}$ & 1,000 \\
\hline$* p<0.05$. & & & \\
$* * p<0.01$. & & & \\
\end{tabular}

a pontuação dos mesmos tanto na Escala de Estratégias de aprendizagem quanto no Teste Cloze.

\section{Discussão}

\section{Potencialidades do jogo "Bingo Melhor Estudante"}

De acordo com o esperado, a sessão de jogo, sem perder o caráter lúdico, atendeu ao primeiro objetivo proposto no planejamento da pesquisa. Mediante as respostas pôde-se conhecer melhor as percepções dos estudantes acerca de comportamentos facilitadores e dificultadores da aprendizagem.

Por outro lado, a análise qualitativa das respostas dos participantes ao Formulário de Avaliação Metacognitiva revelou que nem todos os alunos conseguiram estabelecer uma relação entre a pontuação obtida e o objetivo do jogo, conforme consta no texto das Regras do Jogo lidas e comentadas antes do início da atividade. Foi, entretanto, interessante constatar o percentual dos participantes que reconheceram os benefícios do jogo para sua vida estudantil, ao longo do instrumento, constituindo-se num indicativo preliminar das potencialidades do jogo quanto à possibilidade de desenvolver no participante uma auto-identificação reflexiva e a consciência metacognitiva.

Portanto, pode-se reconhecer a potencialidade do jogo "Bingo Melhor Estudante" como instrumento de avaliação acerca da percepção de alunos sobre as crenças, hábitos, atitudes e estratégias de aprendizagem que caracterizam os bons estudantes. Trata-se, pois, de mais um instrumento à disposição de psicopedagogos e educadores para melhor conhecerem os seus alunos e para melhor trabalharem esse autoconhecimento.

Tendo em vista o hiato que existe entre o relato ou o conhecimento das estratégias de aprendizagem por parte dos alunos e o uso efetivo das mesmas, é importante ressaltar que o jogo BMG deve ser visto como um passo inicial em direção à possibilidade de avaliação da percepção do estudante sobre o que seria um bom aluno - alguém que utiliza estratégias cognitivas e metacognitivas necessárias à aprendizagem auto-regulada. Para que esse instrumento possa vir, de fato, a contribuir para identificar o aprendiz auto-regulado precisa ser utilizado em conjunto com atividades auto-reflexivas sistemáticas e o dado obtido pelo jogo deve ser ainda complementado e confirmado por pesquisas qualitativas que envolvam observações do estudante em atividades de aprendizagem e estudo.

Em linhas gerais, os resultados obtidos podem, pois, ser considerados como promissores, uma vez que o contexto de aplicação do jogo, pelas características e objetivos desse estudo, foi desvinculado do trabalho pedagógico e das demais situações de ensino-aprendizagem, isto é, deve-se considerar que não houve uma continuidade em termos de intervenção psicopedagógica junto aos estudantes.

Os resultados encontrados sugerem possibilidades de novos estudos acadêmicos que poderão ser realizados, visando aperfeiçoar o jogo BME como um dos instrumentos de análise e a Psicologia Cognitiva, baseada na Teoria do Processamento da Informação, como referencial teórico. A seguir serão discutidos os dados que se relacionam ao segundo objetivo desse estudo. 


\section{Relações entre o desempenho dos participantes no jogo, a compreensão da leitura e as estratégias de aprendizagem}

A análise estatística inferencial revelou a existência de uma correlação positiva, moderada e altamente significativa entre o desempenho no jogo e a compreensão na leitura, evidenciando que quanto maior a compreensão em leitura, melhor o desempenho no jogo. A atividade com jogos se constitui, portanto, em oportunidades de utilização da leitura e da escrita de forma contextualizada e, ao mesmo tempo, de uma aproximação entre jogo, leitura e escrita, em estudos futuros.

Na realidade, não existem muitos estudos teóricos focalizando as relações entre jogo e leitura. A partir do referencial teórico pesquisado para tentar desvendar as questões-problema que nortearam este estudo, pode-se inferir alguns eixos de aproximação entre as situações de jogos e as situações de leitura. Entre eles, em primeiro lugar, pode ser considerada a concepção de leitura como uma atividade que, assim como o jogo envolve raciocínio e exige comportamento estratégico (Solé, 1992/1998; Vaz, 1998). Em segundo lugar, entre as estratégias utilizadas pelo educador para desenvolver a aprendizagem auto-regulada por meio dos jogos, pode-se destacar a reconstituição da ação de jogar, por meio da "leitura" dos registros e das notações empregadas no decorrer da partida.

É importante mencionar que também houve correlação positiva e estatisticamente significativa entre os resultados da Escala de Estratégias de Aprendizagem e os resultados do Jogo. Assim, pode-se dizer que médias mais altas na escala associaram-se às melhores pontuações no jogo. Cumpre notar que estudos recentes destacam a importância do trabalho de intervenção em estratégias cognitivas e metacognitivos em diferentes áreas do currículo (Dembo, 2000; Hacker, 1998; Pressley \& Woloshyn, 1995). Mostram também as relações entre a utilização de estratégias e o tornar-se especialista num determinado jogo, assim como as possibilidades de desenvolver estratégias em situações de jogo, no caso de alunos com dificuldades de aprendizagem (Gomes \& Boruchovitch, 2004a; Brenelli, 2001; Macedo \& cols., 2000). Em síntese, o jogo "Bingo Melhor Estudante" parece ser um instrumento que oferece amplas possibilidades de aplicação, embora investigações futuras acerca de suas relações com outras variáveis sejam úteis e necessárias.

\section{Considerações Finais}

O jogo BMG, adaptado para servir como instrumento de avaliação das percepções dos participantes acerca de fatores que favorecem a aprendizagem, atende a uma carência de instrumentos relacionados às estratégias de aprendizagem e à regulação da aprendizagem. Tem a vantagem de assumir uma forma lúdica que favorece o envolvimento dos participantes. Além disso, tem uma dinâmica simples, não depende do fator sorte, nem estimula a competição. Ao contrário, cada jogada exige reflexão e decisão: os participantes precisam comparar procedimentos de aprendizagem e decidir pelos que caracterizam um "bom estudante".

No entanto, como instrumento de possível intervenção, ganhará relevância e sentido a partir de novas investigações que permitam acompanhar possíveis ganhos dos participantes mediante situações criadas pelo pesquisador em função dos objetivos a serem atingidos. Ou seja, mais importante que o jogo por ele mesmo, ou que o simples ato de jogar, é o contexto interacional que se estabelece e a ação estratégica do investigador.

Para preservar essas características no contexto psicopedagógico e educacional, deve-se ter em mente que para o educador o jogo, e cada partida, são, ao mesmo tempo, um objeto de estudo e um meio de ensino e, para o educando, durante a ação de jogar, ocorre a aprendizagem do conteúdo do jogo e dos modos de aprender (Gomes, 2002).

Entre as limitações do presente estudo é preciso considerar, não apenas o pequeno número de participantes, abrangendo uma única turma, de uma das séries do Ensino Fundamental, como também somente a exploração da funcionalidade do "Bingo Melhor Estudante". No entanto, os procedimentos adotados foram suficientes para atingir os objetivos propostos, bem como para se perceber possibilidades de aperfeiçoamento do jogo, enquanto instrumento psicopedagógico, visando um leque mais amplo de aplicação. Aponta-se igualmente, a necessidade de futuros estudos complementares que possam validá-lo, como instrumento de avaliação das percepções dos participantes acerca das características de um bom estudante e para verificar os efeitos das possíveis intervenções com o mesmo.

Vale ressaltar a relevância de estudos que venham suprir as carências na área, e complementar os esforços dos pesquisadores que já estão trabalhando nessa direção. Essa observação refere-se ao jogo "Bingo Melhor Estudante" e a outros instrumentos similares. Propostas de intervenção com estudantes serão úteis e muito necessárias. Em primeiro lugar com alunos, para que se conscientizem sobre o próprio processo de aprendizagem. Em segundo lugar com educadores, para que se tornem estratégicos e auto-regulados no seu trabalho com os estudantes, no desenvolvimento deles quanto ao uso de estratégias de aprendizagem e de ensino, bem como da aprendizagem auto-regulada (Boruchovitch, 1999; Gomes, 2002; Mettrau \& Matias, 1998).

\section{Referências}

Alliende, F. Condemarín M., Chadwick M. \& Milicic N. (1994). Compreensão da Leitura. (J. P dos Santos, Trad.) Campinas, SP: Editorial Psy II. (Trabalho original publicado em 1990).

Almeida, L. S. (1992). Inteligência e aprendizagem: dos seus relacionamentos à sua promoção. Psicologia: Teoria e Pesquisa, 08 (3), 277-292.

Almeida, L. S. (2002). Facilitar a aprendizagem: ajudar os alunos a aprender e a pensar. Psicologia Escolar e Educacional, 6(2), 155-165.

Bardin, L. (1991). Análise de conteúdo. Lisboa: Edições 70, LDA.

Berelson, B. (1952). Content analysis in communications research. Glencoe: Free Press.

Boruchovitch, E. (1999). Estratégias de aprendizagem e desempenho escolar: considerações para a prática educacional. Psicologia: Reflexão e Crítica, 12(2), 361-373.

Boruchovitch, E. (2001a). Algumas estratégias de compreensão em leitura de alunos do Ensino Fundamental. Psicologia Escolare Educacional, 5(1), 19-25. 
Boruchovitch, E. (2001b). Dificuldades de aprendizagem, problemas motivacionais e estratégias de aprendizagem. Em F. F. Sisto, E. Boruchovitch, L. D. T. Fini, R. P. Brenelli \& S. de C. Martinelli (Orgs.), Dificuldades de aprendizagem no contexto psicopedagógico (pp. 40-59). Petrópolis: Vozes.

Boruchovitch, E. (2004a). A auto-regulação da aprendizagem e a escolarização inicial. Em E. Boruchovitch \& J. A. Bzuneck (Orgs.), Aprendizagem - processos psicológicos e o contexto social na escola (pp. 55-88). Petrópolis: Vozes.

Boruchovitch, E. (2004b). Avaliação Psicoeducacional à luz da Psicologia Cognitiva Contemporânea [Resumo]. Anais do X Simpósio de Pesquisa e Intercâmbio Científico da Associação Nacional de Pesquisa e Pós-Graduação em Psicologia (p. 102), Praia Formosa, Aracruz-ES: ANPEPP.

Brenelli, R. P. (2001). Espaço lúdico e diagnóstico em dificuldades de aprendizagens. Em F. F. Sisto, E. Boruchovitch, L. D. T. Fini, R. P. Brenelli \& S. de C. Martinelli (Orgs.), Dificuldades de aprendizagem no contexto psicopedagógico (pp. 167-189). Petrópolis: Vozes.

Bzuneck, J. A. (1999). Uma abordagem sócio-cognitivista à motivação do aluno: a teoria de metas de realização. PsicoUSF, 4(2), 51-66.

Bzuneck, J. A. (2001). As crenças de auto-eficácia e o seu papel na motivação do aluno Em E. Boruchovitch \& J. A. Bzuneck (Orgs.), A motivação do aluno (pp.116-133). Petrópolis: Vozes.

Conselho Nacional de Saúde. Resoluções 196/96 e 251/97. Diretrizes e normas regulamentadoras de pesquisas. Retirado em 24/05/2001. http://conselho.saude.gov.br/docs/Resolucoes/

Couceiro Figueira, A. P. (1997). Aprendizagem auto-regulada: diferentes leituras teóricas. Psychologica, 18, 47-77.

Da Silva, A. L. \& Sá, I. (1993). Saber estudar e estudar para saber. Porto: Porto Editora, Colecção Ciências da Educação.

Dembo, M. H. (1994). Applying educational psychology. (5 $\left.{ }^{\mathrm{a}} \mathrm{ed}.\right)$. New York: Longman.

Dembo, M. H. (2000). Motivation and learning strategies for college success - a self-management approach. London: LEA.

Festas, M. I. F. (1998). A compreensão da leitura: a construção de um modelo mental do texto. Revista Portuguesa de Pedagogia, Ano XXXII(1), 81-98.

Garner, R. \& Alexander, P.A. (1989). Metacognition: Answered and unanswered questions. Educational Psychologist, 24, 143-158.

Gomes, M. A. M. (2002). Aprendizagem auto-regulada em leitura numa perspectiva de jogos de regras. Dissertação de Mestrado, Universidade Estadual de Campinas, Campinas, São Paulo.

Gomes, M. A. M. \& Boruchovitch, E. (2004a). A aprendizagem por meio de jogos: uma abordagem cognitivista. Em E. Boruchovitch \& J. A. Bzuneck (Orgs.), Aprendizagem - processos psicológicos e o contexto social na escola (pp. 89-117). Petrópolis: Vozes.

Gomes, M. A. M. \& Boruchovitch, E. (2004b). Bingo Melhor Estudante: adaptação de um instrumento de diagnóstico da aprendizagem Em C. Machado, L. S. Almeida, M. Gonçalves \& V. Ramalho, X Conferência Internacional Avaliação Psicológica - Formas e Contextos (pp. 539-543). Braga: Psiquilíbrios.

Guthrie, J. T., Wigfield, A. \& Vonsecker, C. (2000). Effects of integrated instruction on motivation and strategy use in reading. Journal of Educational Psychology, 92(2), 331-341.
Hacker, D. J. (1998). Self-Regulated Comprehension During Normal Reading. Em J. D. Hacker, J. Dunlosky \& A. C. Graesser (Orgs.), Metacognition in educational theory and practice (pp. 165-191). London: Associates Publishers (LEA).

Hong, E. \& O'Neil Jr., H. F. (2001). Construct validation of a trait self-regulation model. International Journal of Psychology, 36(3), 186-194.

Macedo, L., Petty, A. L. S. \& Passos, N. C. (2000). Aprender com jogos e situações-problema. Porto Alegre: Artmed.

Mettrau, M. B. \& Mathias, M. T. (1998). O papel social da prática pedagógica do professor na promoção das capacidades sóciocognitivo-afetivas do alunado. Tecnologia Educacional, 26(141), 30-34.

Pozo J. J. (1996). Estratégias de aprendizagem. Em C. Coll, J. Palácios \& A. Marchesi (Orgs.), Desenvolvimento psicológico e educação: Psicologia da Educação (pp. 176-197). Porto Alegre: Artes Médicas.

Pressley, M. \& Woloshyn, V. (1995). Cognitive strategy instruction - that really improves children's academic performance. $\left(2^{\mathrm{a}}\right.$ ed.) Brookline Books.

Quintanal Díaz, J. (1998). Estrategias lectoras. Revista Portuguesa de Educação, 11(1), 123-134.

Sadler, C. R. (2001). Comprehension strategies for meddle grade learners - a handbook for content area teachers. Georgia: International Reading Association.

Solé, I. (1998). Estratégias de leitura. (C. Schilling, Trad.) Porto Alegre: Artmed. (Trabalho original publicado em 1992)

Symons, S., Snyder, B., Cariglia-Bull, T. \& Pressley, M. (1989). Why be optimistic about cognitive strategy instruction? Em C. B. MacCormick, G. Miller \& M. Pressley (Orgs.), Cognitive strategy research: from basic research to educational applications (pp. 1-32). New York: Springer-Verlag.

Valle Arias, A. González Cabanach, R., Rodríguez Martinez S., Piñeiro Aguín, I. \& Suárez Riveiro J. M. (1999). Atribuciones causales, auto concepto y motivación en estudiantes com alto y bajo rendimiento académico. Revista Española de Pedagogía, Año $\operatorname{LVII}(214), 525-546$.

Vauras, M., Kinnunen, R. \& Rauhanummi T. (1999). The role of metacognition in the context of integrated strategy intervention. European Journal of Psychology of Education, XIV(4), 555569.

Vaz, J. P. (1998). Ensinar a compreender: das estratégias de leitura à leitura estratégica. Revista Portuguesa de Pedagogia, XXXII(1), 99-123.

Woolfolk, A. E. (2000). Psicologia da educação. (M. C. Monteiro, Trad.), Porto Alegre: Artmed. (Trabalho original publicado em 1998)

Recebido em 12.02.2005

Primeira decisão editorial em 05.04.2005

Versão final em 09.12.2005

Aceito em 12.12.2005 